\title{
THE CLUSTER-MERGER SHOCK IN 1E 0657-56: FASTER THAN A SPEEDING BULLET?
}

\author{
Miloš Milosavluević, ${ }^{1}$ Jun Koda, ${ }^{1}$ Daisuke Nagai, ${ }^{2}$ Ehud Nakar, ${ }^{2}$ and Paul R. Shapiro ${ }^{1}$ \\ Received 2007 March 8; accepted 2007 April 19; published 2007 May 16
}

\begin{abstract}
Shock waves driven in the intergalactic medium during the merging of galaxy clusters have been observed in $\mathrm{X}$-ray imaging and spectroscopy. Fluid motions inferred from the shock strength and morphology can be compared to the cold dark matter (CDM) distribution inferred from gravitational lensing. A detailed reconstruction of the CDM kinematics, however, must take into account the nontrivial response of the fluid intracluster medium to the collisionless CDM motions. We have carried out two-dimensional simulations of gas dynamics in cluster collisions. We analyze the relative motion of the clusters, the bow shock wave, and the contact discontinuity, and relate these to X-ray data. We focus on the Bullet Cluster, 1E 0657-56, a near-head-on collision of unequalmass clusters, for which the gas density and temperature jumps across the prominent bow shock imply a high shock velocity, $4700 \mathrm{~km} \mathrm{~s}^{-1}$. The velocity of the fluid shock has been widely interpreted as the relative velocity of the CDM components. This need not be the case, however. An illustrative simulation finds that the present relative velocity of the CDM halos is $\sim 16 \%$ lower than that of the shock. While this conclusion is sensitive to the detailed initial mass and gas density profile of the colliding clusters, such a decrease of the inferred halo relative velocity would increase the likelihood of finding $1 \mathrm{E}$ 0657-56 in a $\Lambda \mathrm{CDM}$ universe.
\end{abstract}

Subject headings: dark matter — galaxies: clusters: general — galaxies: clusters: individual (1E 0657-56) intergalactic medium - shock waves $-\mathrm{X}$-rays: galaxies: clusters

\section{INTRODUCTION}

During the growth of structure in the universe, nonlinear CDM halos collide and merge hierarchically. When halos collide, the dark matter of one can, if collisionless, pass through that of the other, unlike the fluid baryonic gas in the halos. Since the collisions are frequently supersonic for this gas, it passes through shocks, where it heats and virializes in the final halo. Currently, an X-ray surface brightness and temperature discontinuity identifiable as a merger shock with Mach number significantly above unity has been detected with Chandra in two galaxy clusters: 1E 0657-56 (Markevitch et al. 2002; Markevitch 2006) and A520 (Markevitch et al. 2005). In both cases, X-ray maps exhibit a bow-shock-like temperature and density jump. For 1E 0657-56, the spatial segregation of the $\mathrm{X}$-ray-emitting plasma from the peaks of the mass distribution detected with gravitational lensing has been interpreted as the first direct proof of the existence of dark matter (Clowe et al. 2006).

The strength and geometry of these shock fronts, and the structure of the contact discontinuity, depend on the kinetic energy and the detailed gravitational and gas density profiles of the merging components. If one could recover the collisionless CDM dynamics from the fluid dynamics of the X-rayemitting gas, then the kinematics of merger shocks would be a powerful probe of the nature and clustering of CDM (e.g., Markevitch et al. 2005; Clowe et al. 2006). However, the two components are coupled only by gravity and behave differently in the merging process. Recently, it has been assumed that the gas shock velocity inferred from the X-ray measurements of 1E 0657-56 equals that of the colliding clusters, implying a rare merger event that might be in conflict with the statistical expectations of the CDM model. We critically examine this

\footnotetext{
${ }^{1}$ Department of Astronomy, University of Texas, 1 University Station, C1400, Austin, TX 78712.

${ }^{2}$ Theoretical Astrophysics, California Institute of Technology, Mail Code 130-33, 1200 East California Boulevard, Pasadena, CA 91125.
}

assumption by simulating gas dynamics in cluster mergers and studying the response of the intergalactic medium (IGM).

In the cluster merger in $1 \mathrm{E} 0657-56,{ }^{3}$ transverse motion of the merger shock $\left(\approx 4700 \mathrm{~km} \mathrm{~s}^{-1}\right.$; Markevitch 2006$)$ greatly exceeds the relative radial motion of galaxies in the two components $\left(\approx 600 \mathrm{~km} \mathrm{~s}^{-1}\right.$; Barrena et al. 2002). This suggests that the shock was driven in the IGM of the larger component by a near-head-on supersonic passage of the smaller component. Inside the bow shock, another bow-shaped discontinuity with a reverse temperature jump (lower temperature on the convex side) is seen; this has been interpreted as the contact discontinuity separating the shocked and ram pressure stripped IGM of the two merging components. Takizawa $(2005,2006)$ recovered the three basic features (a bow shock, a contact discontinuity, and ram pressure stripping) in hydrodynamical simulations of cluster collisions, but did not address the relative kinematics of the CDM and the fluid component.

We study the evolution of the merger shock wave and the contact discontinuity, and relate these observable features of X-ray maps to CDM kinematics. In $\S 2$ we describe the numerical simulations and analyze a particular run that approximates the observed 1E 0657-56 (the conclusions also apply to other cluster mergers). In $\S 3$ we discuss implications for CDM clustering. The standard cosmological parameters consistent with the Wilkinson Microwave Anisotropy Probe (WMAP; Spergel et al. 2007) are assumed throughout.

\section{SIMULATIONS OF CLUSTER MERGER SHOCKS}

\subsection{The Algorithm and Initial Conditions}

The simulations were carried out with the thoroughly tested Eulerian code ASC FLASH (Fryxell et al. 2000) in two spatial dimensions. Its adaptive mesh-refinement capability allowed us to simulate a spatial domain with dimensions of $20 \mathrm{Mpc}$ and

\footnotetext{
${ }^{3}$ With an average X-ray temperature of $14.1 \pm 0.2 \mathrm{keV}$ (Markevitch et al. 2002; Markevitch 2006; Andersson et al. 2006; Markevitch \& Vikhlinin 2007), the cluster 1E 0657-56 at redshift $z=0.296$ (Tucker et al. 1998) is the hottest and X-ray-brightest known cluster.
} 


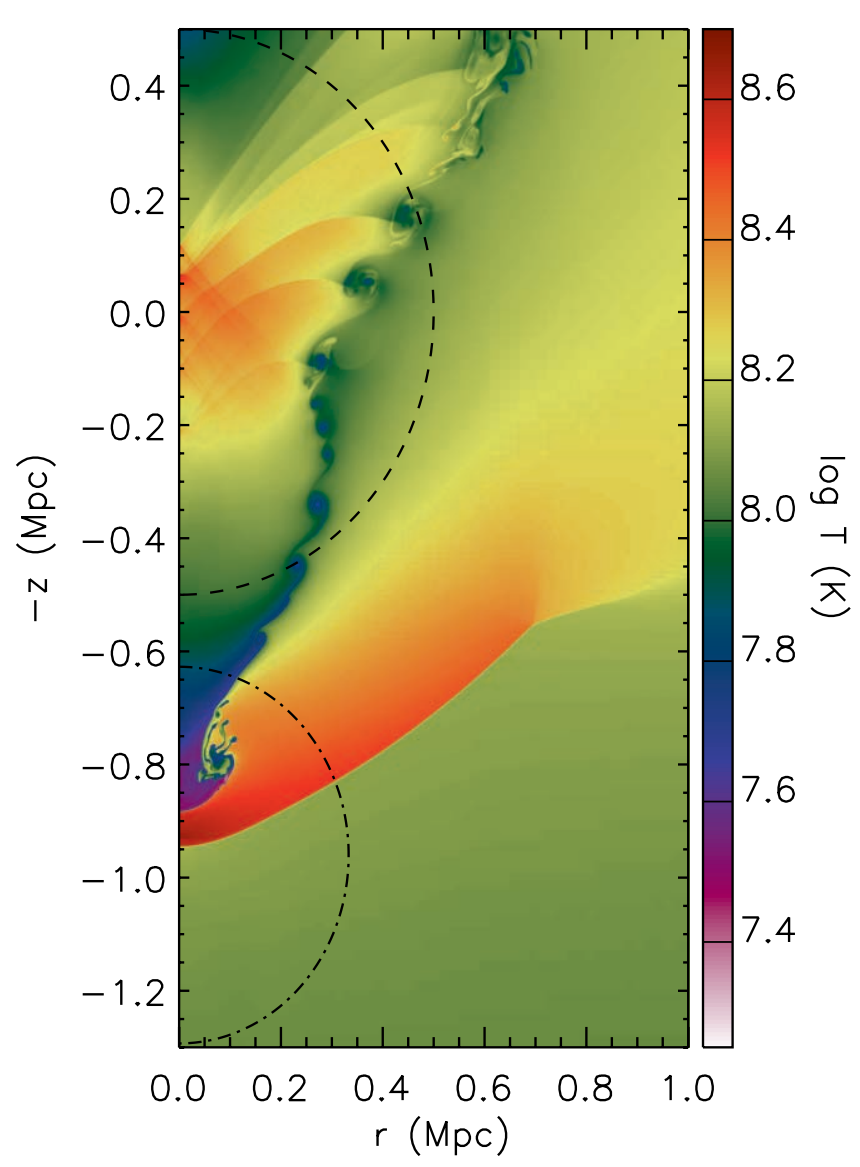

Fig. 1.-Temperature in the meridional plane after cluster pericenter passage. The larger CDM halo is located at $(r, z)=(0,0)$. The circles indicate the scale radii $r_{s}$ of the halos.

simultaneously achieve a spatial resolution of $\sim 1 \mathrm{kpc}$ at fluid discontinuities. The simulations were carried out in cylindrical $(r, z)$ coordinates in an inertial frame. Axial symmetry restricts the simulations to head-on cluster collisions. To avoid artifacts that can result when a perfectly head-on collision occurs with cuspy halo profiles and to approximate the conditions in nonhead-on collisions, the central dark matter and gas density of the larger cluster were artificially reduced. The gravitational potential of each of the clusters is spherically symmetric and a fixed function of distance from the center. The clusters are placed at separation $D_{12}$ and are allowed to move under each other's gravity. The acceleration of each cluster is evaluated at its center. The effect of the Hubble flow on cluster motion is ignored because it only affects the early infall, while we are interested in the dynamics of the two halos and their gas content at pericenter passage. We also ignore the dynamical friction drag that reduces the halo velocity during pericenter passage. The fluid flow and its relation to CDM dynamics within a few hundred kiloparsecs from the center of the smaller halo should not be different in the presence of dynamical friction, because the gravitational field of the dynamical wake that trails the smaller CDM halo will not have strong gradients (tides) on such scales. Finally, we ignore the tidal stripping of the smaller CDM halo, which could be substantial on larger scales than are relevant here.

For the mass distribution associated with the gravitational potential, we consider the Navarro et al. (1997) profile (NFW), $\rho_{\mathrm{NFW}}=\rho_{0}\left(r / r_{s}\right)^{-1}\left(1+r / r_{s}\right)^{-2}$, and the "core" profile $\rho_{\text {core }}=$ $\rho_{0}\left(1+r / r_{s}\right)^{-3}$. The profiles were normalized such that the mass enclosed within a fixed radius $r_{500}$ equals the mass of the cluster $M_{500}$. The gas density profile was allowed to differ from that of the CDM profile. At the start of each simulation, monatomic gas with an adiabatic polytropic equation of state $(\gamma=5 / 3)$ is set in hydrostatic equilibrium within each halo. Explicit viscosity, heat conduction, cooling, and self-gravity of the gas are ignored. Runs were carried out for a variety of merger velocities and density profiles that included NFW and core CDM profiles, as well as cuspy (divergent) and noncuspy gas density profiles. In divergent CDM density profiles, a small constant-density core $(20 \mathrm{kpc})$ in hydrostatic equilibrium was applied in the center to resolve the fluid gradients with multiple resolution elements. The cluster gas fraction was $f_{\mathrm{gas}} \Omega_{b} / \Omega_{m}$, where $\Omega_{b} / \Omega_{m} \approx 0.16$ is the cosmological baryon abundance and $f_{\text {gas }} \approx 0.7$ (Afshordi et al. 2007). The mean molecular weight was $\mu=0.59$, corresponding to $75 \%$ hydrogen and $25 \%$ helium by mass, fully ionized. Numerical convergence was ascertained by resimulation at increased spatial resolution.

\subsection{General Features of Cluster Merger Shocks}

Two prominent features seen in all simulations are a bow shock wave and a contact discontinuity (cold front). The formation of a shock wave during a cluster merger was discussed by Markevitch et al. (2000); our results are consistent with their picture. The opening angle and the radius of curvature of the nose of the bow shock are sensitive to the details of the simulation, but both are larger than those expected in steady state bow shocks driven by spherical hard spheres moving with a constant velocity in a uniform medium (e.g., Farris \& Russell 1994; Vikhlinin et al. 2001 and references therein). Dense gas on the convex side of the contact discontinuity originates in the smaller cluster and remains cold throughout the collision. The wings of the contact discontinuity are Kelvin-Helmholtz $(\mathrm{KH})$ unstable (in real clusters, the KH instability will be modified by departures from ideal hydrodynamics). A nonlinear instability disrupts the coherence of the surface of the discontinuity and creates a narrowing in its axial diameter (a neck) just behind the nose, giving it a mushroom-like or bullet-like appearance (see Fig. 1). At later times (not shown), the Rayleigh-Taylor instability eats into and disrupts the bullet from the rear, as predicted by Markevitch \& Vikhlinin (2007).

In most simulations, during pericenter passage, the shock and contact discontinuities are slower than the relative velocities of the two CDM halos (shock velocity is calculated in the local rest frame on the upstream side of the shock). This is due to the ram pressure force acting on the gas in the center of the smaller cluster but not on the halo. In many runs, some degree of ram pressure stripping is evident in the center of the smaller cluster (the smaller cluster is "stripped" when cold gas originating in the smaller cluster has been expelled from the center of the smaller halo). However, later, the halos climb out of each other's gravitational potential well and decelerate, but the shock and the contact discontinuity do not decelerate appreciably over a longer period (Fig. 2a). The lack of deceleration of the shock and the cold front was noticed, although not quantified, in previous simulations of cluster mergers (Markevitch \& Vikhlinin 2007 and references therein). It could result from the drop in ram pressure as the cold bullet propagates into a thinning larger cluster's atmosphere and from the gravitational tide of the small cluster's halo. After the cold gas originally in the smaller cluster has been ram pressure stripped, the stripped gas lags behind the smaller halo, but eventually its velocity exceeds that of the halo, which implies that the gas can later catch up with the halo again. The shock kinematics and morphology are 


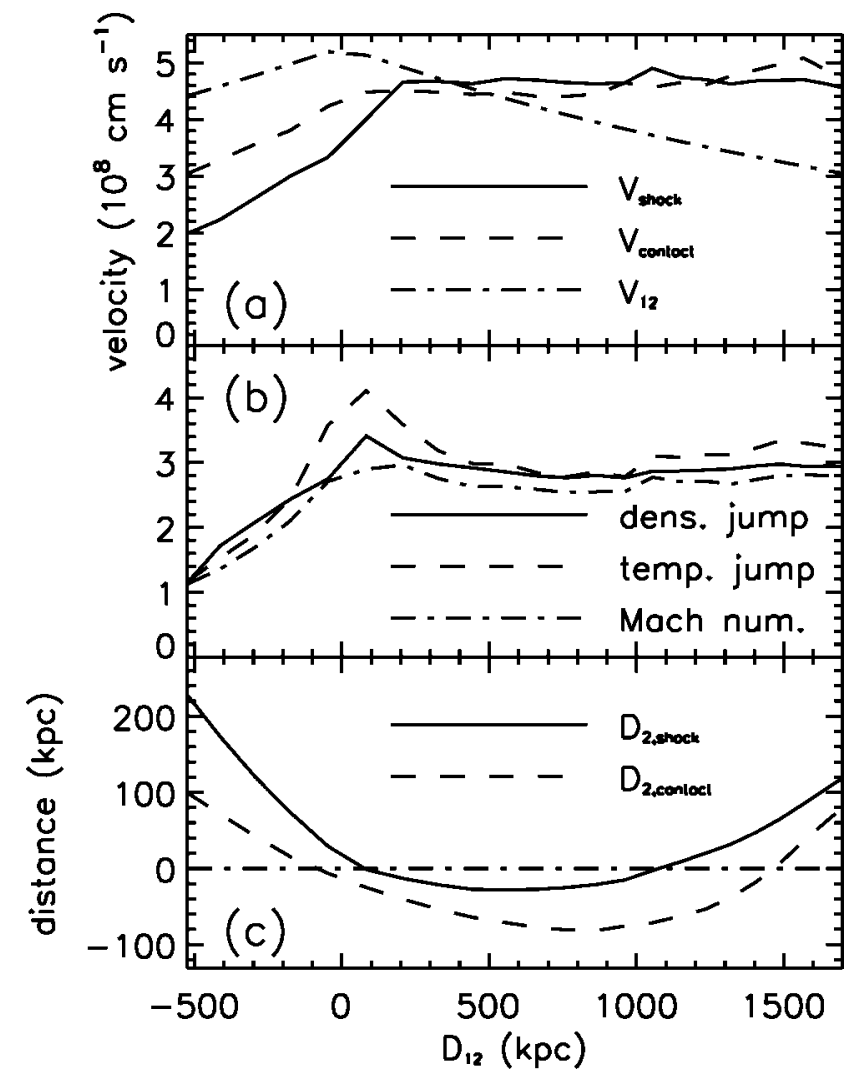

FIG. 2.- (a) Velocity of the shock (solid line) and of the contact discontinuity (dashed line) relative to the preshock gas upstream, and the relative velocity of the CDM halos (dot-dashed line), all as functions of the time-varying separation between the halos, $D_{12}=z_{2}-z_{1}$. (b) Density (solid line) and temperature jump (dashed line) at the shock, and the shock Mach number (dotdashed line). (c) Distance of the shock (solid line) and the contact discontinuity (dashed line) from the center of the smaller halo.

extraordinarily sensitive to the parameters of the simulation. A small variation $(\sim 25 \%)$ in the cluster mass ratio or density profile can lead to vastly different degrees of ram pressure stripping, or the opening angle of the bow shock.

\subsection{A Model of 1E 0657-56}

General results discussed in $\S 2.2$ are illustrated by a specific run that came the closest to reproducing the observed properties of 1E 0657-56. Cluster masses in the run were $M_{500,1}=$ $1.27 \times 10^{15} M_{\odot}$ and $M_{500,2}=2.54 \times 10^{14} M_{\odot}$, cluster radii were $r_{500,1}=1.5 \mathrm{Mpc}$ and $M_{500,2}=1 \mathrm{Mpc}$, and the cluster scale radii were $r_{s, 1}=500 \mathrm{kpc}$ and $r_{s, 2}=333 \mathrm{kpc}$. ${ }^{4}$ The density profile of the larger cluster was the nondivergent $\rho_{\text {core }}$ with central density $\approx 2.3 \times 10^{-3} M_{\odot} \mathrm{pc}^{-3}$ (again, to approximate

\footnotetext{
${ }^{4}$ The mass ratio of the two halos in the simulation, $M_{500,1} / M_{500,2}=5$, is about half of the ratio of $\sim 10$ inferred from lensing data (Clowe et al. 2004, 2006; Bradač et al. 2006). Some fraction of the smaller cluster's initial mass will have been tidally stripped during the merger, possibly reconciling our initial conditions with lensing. The total luminosity $L_{\text {tot }}$ of galaxies in each cluster can be used to estimate the cluster mass via the relation $L_{\text {tot }} \propto M_{500}^{\beta}$ (Lin et al. 2004; Miller et al. 2005; Cooray \& Milosavljević 2005), where $\beta \lesssim 1$ is a bandpass-dependent factor. For the $R$ band we adopt $\beta_{R}=0.65$. Barrena et al. (2002) reported $L_{\text {tot, } 1, R}=10^{12} L_{\odot}$ and $L_{\text {tot, } 2, R}=0.2 \times 10^{12}$ $L_{\odot}$, implying a mass ratio of $M_{500,1} M_{500,2} \approx 12$, in agreement with lensing. The difference of the mass ratios may also be attributed to a slightly nonhead-on collision in 1E 0657-56. Indeed, the lensing maps are consistent with a northerly passage of the smaller cluster inducing the observed southwestnortheast tidal distortion in the larger cluster. Then, the lower density column met by the small cluster would result in equivalent shock dynamics if the smaller cluster were smaller than in the simulation.
}
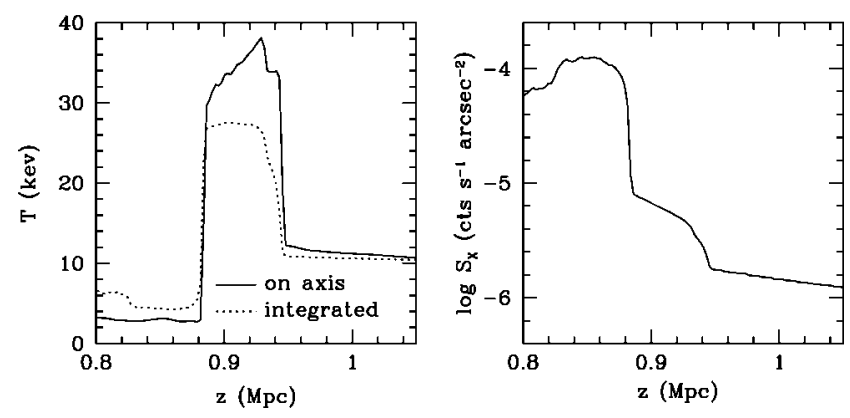

FIG. 3.-Temperature profile on the central axis (left panel, solid line), emissivity-weighted integrated temperature profile (left panel, dotted line), and 0.8-4 keV surface brightness on the central axis (right panel). The center of the larger CDM halo is located at $z=0$. The bow shock at $z \sim 0.94 \mathrm{Mpc}$ is split into a main shock and a weaker subshock.

the conditions in a non-head-on merger with pericenter passage at a distance of $\sim r_{s, 1}$ ), and, in the smaller cluster, it was the NFW profile $\rho_{\mathrm{NFW}}$. The gas density in both clusters was proportional to the CDM density, $\rho_{\text {gas }}=\left(1+\Omega_{m} / f_{\text {gas }} \Omega_{b}\right)^{-1} \rho_{\mathrm{CDM}}$. The halos initiated infall from relative rest at separation $D_{12}=4.6 \mathrm{Mpc}$, and their maximum relative velocity at pericenter was $V_{\max } \approx 5270 \mathrm{~km} \mathrm{~s}^{-1}$.

Figure 1 shows a snapshot of the meridional temperature distribution when the distance of the shock to the larger CDM halo is about $1 \mathrm{Mpc}$. The larger and smaller clusters are moving vertically upward and downward in the figure, respectively. The cold gas bullet is $\sim 100 \mathrm{kpc}$ in radius, excluding visible fingers of cold gas embedded in the hot postshock medium. The center of the smaller halo is located ahead (below) the contact discontinuity, and almost exactly at the location of the bow shock, as found in X-ray and lensing maps of 1E 0657-56 (Clowe et al. 2004, 2006; Bradač et al. 2006). This is also evident in Figure $2 c$, which shows the position of the bow shock and the contact discontinuity relative to the position of the smaller halo. Ram pressure stripping at the center of the smaller cluster occurs at $D_{12}=0$, but the bow shock and the contact discontinuity catch up with the halo at $D_{12} \approx 1.2$ and $\approx 1.5 \mathrm{Mpc}$, respectively.

The preshock and postshock axial temperatures are 12 and $34 \mathrm{keV}$, respectively (see Fig. 3). Figure $2 b$ shows the evolution of the density and temperature jumps across the shock, and its Mach number, as a function of the halo separation. The Mach number is $\approx 3$ when the separation equals the projected separation of lensing-density peaks $D_{12} \approx 720 \pm 25 \mathrm{kpc}$ (Clowe et al. 2004, 2006; Bradač et al. 2006). The temperatures and shock strength are compatible with the results of Markevitch (2006), who, for a $\gamma=5 / 3$ polytropic equation of state and instant electron-ion equilibration, derived the Mach number from deprojected density and temperature jumps in the bow shock in 1E 0657-56. The separation of the shock and the contact discontinuity is $\sim 70 \mathrm{kpc}$, somewhat smaller than in the observed system. Figure 4 shows the predicted X-ray surface brightness map for the simulated cluster.

Figure $2 a$ shows the evolution of the velocities of the shock, the contact discontinuity, and the relative velocity of the two CDM halos. While the cluster velocity decreases as the small halo climbs the potential of the larger halo, the shock velocity remains constant. The lack of deceleration of the shock can be

\footnotetext{
${ }^{5}$ All dimensionless numbers (e.g., the shock Mach number, the gas-to-dark matter velocity ratio) and distances (e.g., the radius of curvature of the shock) are invariant under a uniform scaling of the CDM density that preserves lengths. The scaling preserves the mass-to-temperature ratio.
} 


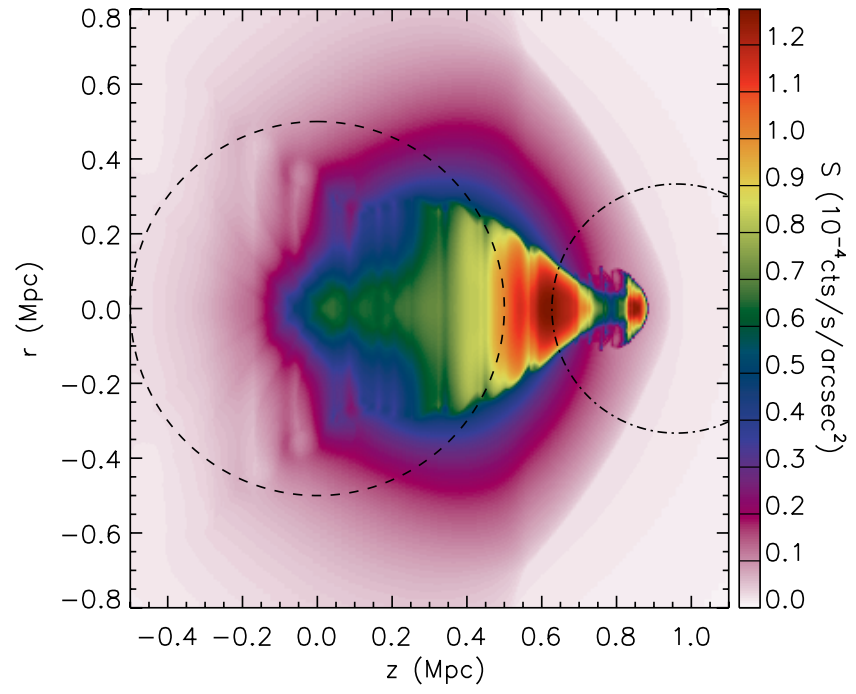

FIG. 4.- Surface brightness map of the simulated Bullet Cluster in the 0.8-4 keV band, assuming a frequency-independent effective area of $400 \mathrm{~cm}^{2}$.

explained by a combination of factors; in addition to gravitational forces, the negative density and temperature gradients into which the shock propagates tend to increase its strength. At $D_{12}=$ $720 \mathrm{kpc}$, the velocity of the shock is $V_{\mathrm{sh}} \approx 4800 \mathrm{~km} \mathrm{~s}^{-1}$, consistent with $V_{\mathrm{sh}} \sim 4740_{-550}^{+710} \mathrm{~km} \mathrm{~s}^{-1}$ inferred for 1E 0657-56 by M. Markevitch (private communication, as cited in Farrar \& Rosen 2007, hereafter FR07). However, the relative velocity of the halos in our simulation is much less, $V_{\text {sub }} \sim 4050 \mathrm{~km} \mathrm{~s}^{-1}$.

\section{DISCUSSION AND CONCLUSIONS}

Recent analyses of 1E 0657-56 (e.g., Hayashi \& White 2006, hereafter HW06; Clowe et al. 2006; Bradač et al. 2006; FR07) assume that the shock velocity equals the instantaneous relative velocity of the CDM halos; note that it is much larger than the virial velocity of the larger cluster. HW06 and FR07 estimated the probability that a halo in the $\Lambda$ CDM universe has a subhalo with a velocity larger than that of the bow shock in 1E $0657-56$. We assess a correction that should be applied to the inferred probabilities in order to account for the relative motion of the shock and the subhalo. HW06 calculated the likelihood of finding a halo-subhalo pair with such relative

\footnotetext{
${ }^{6}$ If the true halo separation is larger than the projected separation of $720 \mathrm{kpc}$, the predicted subcluster velocity $V_{\text {sub }}$ will be $<4050 \mathrm{~km} \mathrm{~s}^{-1}$.
}

velocity by extrapolating results of the Millennium cosmological $N$-body simulation. They find that it decreases rapidly with $V_{\text {sub }}, \log \left[N_{1}\left(>V_{\text {sub }}\right) / N_{\text {hosts }}\right]=-\left(V_{\text {sub }} / v_{10} V_{200}\right)^{\alpha}$, where $v_{10} \approx 1.55$ and $\alpha \approx 3.3$. The probability depends on the assumed values of $V_{\text {sub }}$ and $V_{200}$, in which HW06 and FR07 differ. ${ }^{7}$ HW06 adopted a shock velocity of $V_{\mathrm{sh}} \approx 4500_{-800}^{+1100} \mathrm{~km} \mathrm{~s}^{-1}$ (Markevitch et al. 2004) as the relative velocity $V_{\text {sub }}$ of the two halos and adopted $V_{200} \approx 2380 \mathrm{~km} \mathrm{~s}^{-1}$ for the virial velocity. With these, they infer that the probability of the most massive subhalo having such a velocity is $N_{1}\left(>V_{\text {sub }}\right) \approx 1 \%$, whereas when they adopt a lower shock velocity consistent with uncertainties of $V_{\text {sub }}=3700 \mathrm{~km} \mathrm{~s}^{-1}$, the probability is $N_{1}\left(>V_{\text {sub }}\right) \approx 10 \%$. In our simulation $(\S 2.3)$, we find that when the halos are separated by the observed projected distance $D_{12}=720 \mathrm{kpc}$, the velocity of the shock and that of the subhalo differ by $\left(V_{\mathrm{sh}}-\right.$ $\left.V_{\text {sub }}\right) / V_{\text {sub }} \approx 16 \%$. Thus, the probabilities reported by HW06 should be corrected and should read $\approx 8 \%$ and $\approx 27 \%$, respectively. FR07 adopted a somewhat higher shock velocity, $V_{\mathrm{sh}} \approx 4740 \mathrm{~km} \mathrm{~s}^{-1}$, and a significantly lower virial velocity, $V_{200} \approx 1740 \mathrm{~km} \mathrm{~s}^{-1}$. These values imply a very low probability of $\approx 3.7 \times 10^{-7} .{ }^{8}$ The probability is significantly higher but remains very low, $\approx 2.4 \times 10^{-4}$, when the $16 \%$ correction for the difference between the shock velocity and CDM halo velocity is taken into account.

In conclusion, our two-dimensional hydrodynamical simulations of merging galaxy clusters show that in a case such as the Bullet Cluster 1E 0657-56, the halo collision velocity need not be the same as the intergalactic gas shock velocity. While the kinematics of the shock is sensitive to the details of the cluster structure, the instantaneous shock velocity can exceed the relative velocity of CDM halos by at least $\sim 16 \%$. Any attempt to relate shock kinematics to the details of hierarchical clustering and halo assembly must take into account the nontrivial response of the IGM. Published estimates of the likelihood of finding a configuration resembling 1E 0657-56 in a $\Lambda \mathrm{CDM}$ universe may require upward revision by a factor of 2-700.

Research was supported in part by the Sherman Fairchild Foundation and NASA Astrophysical Theory Program grant NNG 04-G177G. The software used in this work was developed in part by the DOE-supported ASC/Alliance Center for Astrophysical Thermonuclear Flashes at the University of Chicago.

\footnotetext{
${ }^{7}$ The lensing data suggest that HW06's value of $V_{200}$ is an overestimate.

${ }^{8}$ HW06 and FR07 also reported 5 times lower probabilities after counting only postcollision systems.
}

\section{REFERENCES}

Afshordi, N., Lin, Y.-T., Nagai, D., \& Sanderson, A. J. R. 2007, MNRAS, in press (astro-ph/0612700)

Andersson, K. E., Peterson, J. R., \& Madejski, G. M. 2006, ApJ, submitted (astro-ph/0612038)

Barrena, R., Biviano, A., Ramella, M., Falco, E. E., \& Seitz, S. 2002, A\&A, 386,816

Bradač, M., et al. 2006, ApJ, 652, 937

Clowe, D., Bradač, M., Gonzalez, A. H., Markevitch, M., Randall, S. W., Jones, C., \& Zaritsky, D. 2006, ApJ, 648, L109

Clowe, D., Gonzalez, A., \& Markevitch, M. 2004, ApJ, 604, 596

Cooray, A., \& Milosavljević, M. 2005, ApJ, 627, L85

Farrar, G. R., \& Rosen, R. A. 2007, Phys. Rev. Lett., in press (astro-ph/ 0610298) (FR07)

Farris, M. H., \& Russell, C. T. 1994, J. Geophys. Res., 99, 17681

Fryxell, B., et al. 2000, ApJS, 131, 273

Hayashi, E., \& White, S. D. M. 2006, MNRAS, 370, L38 (HW06)

Lin, Y.-T., Mohr, J. J., \& Stanford, S. A. 2004, ApJ, 610, 745
Markevitch, M. 2006, in Proc. X-Ray Universe 2005, ed. A Wilson (ESA SP604; Noordwijk: ESA), 723

Markevitch, M., Gonzalez, A. H., Clowe, D., Vikhlinin, A., Forman, W., Jones, C., Murray, S., \& Tucker, W. 2004, ApJ, 606, 819

Markevitch, M., Gonzalez, A. H., David, L., Vikhlinin, A., Murray, S., Forman, W., Jones, C., \& Tucker, W. 2002, ApJ, 567, L27

Markevitch, M., Govoni, F., Brunetti, G., \& Jerius, D. 2005, ApJ, 627, 733

Markevitch, M., \& Vikhlinin, A. 2007, Phys. Rep., in press (astro-ph/0701821)

Markevitch, M., et al. 2000, ApJ, 541, 542

Miller, C. J., et al. 2005, AJ, 130, 968

Navarro, J. F., Frenk, C. S., \& White, S. D. M. 1997, ApJ, 490, 493

Spergel, D. N., et al. 2007, preprint (astro-ph/0603449)

Takizawa, M. 2005, ApJ, 629, 791 2006, PASJ, 58, 925

Tucker, W., et al. 1998, ApJ, 496, L5

Vikhlinin, A., Markevitch, M., \& Murray, S. S. 2001, ApJ, 551, 160 